PROCEEDINGS OF THE AMERICAN MATHEMATICAL SOCIETY

Volume 126, Number 3, March 1998, Pages 841-851

S 0002-9939(98)04118-5

\title{
CROSSED PRODUCTS OF HILBERT C*-BIMODULES BY COUNTABLE DISCRETE GROUPS
}

\author{
TSUYOSHI KAJIWARA AND YASUO WATATANI
}

(Communicated by Palle E. T. Jorgensen)

\begin{abstract}
We introduce a notion of crossed products of Hilbert $\mathrm{C}^{*}$-bimodules by countable discrete groups and mainly study the case of finite groups following Jones index theory. We give a sufficient condition such that the crossed product bimodule is irreducible. We have a bimodule version of Takesaki-Takai duality. We show the categorical structures when the action is properly outer, and give some example of this construction concerning the orbifold constructions.
\end{abstract}

\section{INTRODUCTION}

The notion of strong Morita equivalence on $\mathrm{C}^{*}$-algebra was introduced by M. Rieffel [Ri1], [Ri2], [Ri3]. It is described by the existence of an imprimitivity bimodule which gives an isomorphism of K-groups.

On the other hand V. Jones [Jo] initiated subfactor theory and the classification of subfactors of a hyperfinite $I I_{1}$-factor with index less than four was obtained in Ocneanu [Oc] and Popa [Po2]. Its development shows importance of bimodules for von Neumann algebras (i.e. correspondences introduced by A. Connes).

In our previous paper $[\mathrm{KW}]$ we were led to the notion of Hilbert $\mathrm{C}^{*}$-bimodules by both strong Morita equivalences and subfactor theory. In the theory of Hilbert $\mathrm{C}^{*}$-bimodule, the associativity condition of two sided inner products in imprimitivity bimodules is replaced by Pimsner-Popa type inequality [PP]. The index of Hilbert $\mathrm{C}^{*}$-bimodules measures the failure from imprimitivity bimodules. Moreover a Hilbert $\mathrm{C}^{*}$-bimodule ${ }_{A} X_{B}$ of finite type represents an element of Kasparov groups [Kas] $\mathrm{KK}(A, B)$ and gives homomorphisms between K-groups $\mathrm{K}_{*}(A)$ and $\mathrm{K}_{*}(B)$. See also [Wa] for the basic fact on index theory for simple $C^{*}$-subalgebras.

In this paper, we shall consider actions on Hilbert $\mathrm{C}^{*}$-bimodules from two kinds of motivations. One is the equivariant Morita equivalence studied by F. Combs [Co], Curto-Muhly-Williams [CMW] and the first named author [Kaj]. It implies strong Morita equivalence of crossed products. See also equivariant K-theory by G. G. Kasparov [Kas] and the equivariant Brauer groups studied by CrockerKumjian-Raeburn-Williams [KRW], [CKRW].

The other motivation is the automorphisms for subfactors. Y. Kawahigashi [Kaw] introduced the orbifold construction in subfactor theory to show that there exist automorphisms of period two on subfactors with principal graph $\mathrm{A}_{4 n-3}$ such

Received by the editors May 15, 1996 and, in revised form, September 10, 1996.

1991 Mathematics Subject Classification. Primary 46L05, 46L37, 46L55.

(C)1998 American Mathematical Society 
that the simultaneous fixed point subalgebras give subfactors with principal graph $\mathrm{D}_{2 n}$. See also M. Choda [Ch] and Y. Katayama [Kat] for the duality of graphs. These automorphisms are non-strongly outer action of Choda-Kosaki [CK] or nonproperly outer action of S. Popa [Po1]. Non-strongly outer automorphism $\alpha \in$ Aut $M$ is characterized by that the corresponding bimodules $M \alpha$ appear in the principal graph. But these automorphisms are not necessary to preserve the subfactor globally. The class of such automorphisms are studied in M. Izumi [I1], [I2], Kosaki [Ko1], [Ko2], S. Goto [Go] and S. Yamagami [Y]. The restriction of these automorphisms to invariant submodules provide many examples of actions on Hilbert $\mathrm{C}^{*}$-bimodules in this paper.

We consider crossed products of Hilbert $\mathrm{C}^{*}$-bimodules and give a sufficient condition which guarantees the irreducibility of the crossed product. We also have Takesaki-Takai duality for Hilbert $\mathrm{C}^{*}$-bimodules. S. Yamagami $[\mathrm{Y}]$ establishes an abstract duality in tensor category. we take a rather concrete approach.

The authors express their hearty thanks to S. Yamagami for several discussions and sending us new preprints about these matters.

\section{Definitions}

We review the definition of Hilbert $\mathrm{C}^{*}$-bimodules of finite type following $[\mathrm{KW}]$. We refer the definition of Hilbert $\mathrm{C}^{*}$-module to [Bl]. Let $A$ and $B$ be unital $\mathrm{C}^{*}$ algebras. Let $X$ be a $\mathbf{C}$-vector space.

Definition $1.1([\mathrm{KW}]), X$ is called a Hilbert $C^{*}$-bimodule (or Hilbert $A$ - $B$ bimodule) if the following conditions hold.

(1) $X$ is a left Hilbert $A$-module.

(2) $X$ is a right Hilbert $B$-module.

(3) Left $A$ action and right $B$ action commute each other.

(4) Let $\lambda(a) x=a x, \rho(b) x=x b$ for $a \in A, b \in B$ and $x \in X$. Then $\lambda(a)$ is bounded and has an adjoint with respect to $\langle,\rangle_{B}$, and $\rho(b)$ is bounded and has an adjoint with respect to ${ }_{A}\langle$,$\rangle .$

(5) Two norms on $X$ given by ${ }_{A}\|x\|=\left\|{ }_{A}\langle x, x\rangle\right\|^{1 / 2}$ and $\|x\|_{B}=\left\|\langle x, x\rangle_{B}\right\|^{1 / 2}$ are equivalent (Pimsner-Popa type inequality).

Definition $1.2([\mathrm{KW}])$. A Hilbert $A$ - $B$ bimodule $X$ is called of finite type if the following two conditions hold.

(1) There exists a finite subset $\left\{u_{i}\right\}_{i}$ in $X$ such that $\sum_{i} u_{i}\left\langle u_{i}, x\right\rangle_{B}=x$ for all $x \in X$.

(2) There exists a finite subset $\left\{v_{j}\right\}_{j}$ in $X$ such that $\sum_{j}{ }_{A}\left\langle x, v_{j}\right\rangle v_{j}=x$ for all $x \in X$.

We call the subset $\left\{u_{i}\right\}_{i}$ the right $B$-basis of $X$ and $\left\{v_{j}\right\}_{j}$ the left $A$-basis of $X$. Moreover we put the right index and left index by r-Ind $[X]=\sum_{i}{ }_{A}\left\langle u_{i}, u_{i}\right\rangle$ and l-Ind $[X]=\sum_{j}\left\langle v_{j}, v_{j}\right\rangle_{B}$.

The following lemma is convenient for verifying the axioms of Hilbert $\mathrm{C}^{*}$ bimodules, because analytic properties follow from purely algebraic properties under the existence of bases.

Lemma $1.3([\mathrm{KW}])$. Let a complex vector space $X$ satisfy the following (1)-(10).

(1) $X$ is a left $A$-module.

(2) $X$ has a left self adjoint (not necessarily positive) $A$-inner product ${ }_{A}\langle\cdot, \cdot\rangle$. 
(3) ${ }_{A}\langle a x, y\rangle=a_{A}\langle x, y\rangle$.

(4) $X$ is a right $B$-module.

(5) $X$ has a right self adjoint (not necessarily positive) $B$-inner product $\langle\cdot, \cdot\rangle_{B}$.

(6) $\langle x, y b\rangle_{B}=\langle x, y\rangle_{B} b$.

(7) Left $A$ action and right $B$ action commute each other.

(8) $\langle a x, y\rangle_{B}=\left\langle x, a^{*} y\right\rangle_{B}$ and ${ }_{A}\langle x, y b\rangle={ }_{A}\left\langle x b^{*}, y\right\rangle$.

(9) There exists a finite subset $\left\{u_{i}\right\}_{i}$ in $X$ such that $\sum_{i} u_{i}\left\langle u_{i}, x\right\rangle_{B}=x$ for all $x \in X$.

(10) There exists a finite subset $\left\{v_{j}\right\}_{j}$ in $X$ such that $\sum_{j}{ }_{A}\left\langle x, v_{j}\right\rangle v_{j}=x$ for all $x \in X$.

Then the other properties in Definition 1.1 are automatically satisfied, and $X$ becomes a Hilbert $C^{*}$-bimodule of finite type.

For $x, y \in X$, we put a (right) rank one operator by $\Theta_{x, y} z=x\langle y, z\rangle_{B}$ for each $z \in X$. We denote by $\mathbf{K}_{B}\left(X_{B}\right)$ the norm closure of the set of linear combinations of (right) rank one operators. Similarly we define ${ }_{A} \mathbf{K}\left({ }_{A} X\right)$.

We shall introduce the crossed product of Hilbert $\mathrm{C}^{*}$-bimodule by a countable discrete group. Throughout the paper we always assume that $A$ and $B$ are unital $\mathrm{C}^{*}$-algebras and $X={ }_{A} X_{B}$ is a Hilbert $\mathrm{C}^{*}$-bimodule of finite type. Let $G$ be a countable discrete group, $\alpha$ be an action of $G$ on $A, \beta$ be an action of $G$ on $B$ and $\gamma$ be a homomorphism from $G$ to the isometry group of $X$ with respect to both norms $_{A}\|\cdot\|$ and $\|\cdot\|_{B}$.

Definition 1.4. Under the above situation, the system $(X, A, B, G, \gamma, \alpha, \beta)$ is called a $G$-equivariant system if the following hold for all $g \in G$ :

$$
\begin{aligned}
\alpha_{g}\left({ }_{A}\langle x, y\rangle\right) & ={ }_{A}\left\langle\gamma_{g}(x), \gamma_{g}(y)\right\rangle, & & \gamma_{g}(a x)=\alpha_{g}(a) \gamma_{g}(x), \\
\beta_{g}\left(\langle x, y\rangle_{B}\right) & =\left\langle\gamma_{g}(x), \gamma_{g}(y)\right\rangle_{B}, & & \gamma_{g}(x b)=\gamma_{g}(x) \beta_{g}(b)
\end{aligned}
$$

for $x, y \in X, a \in A, b \in B$.

First we consider the case where $G$ is a finite group. Let

$$
A \rtimes_{\alpha} G=\left\{\sum_{g \in G} a_{g} u_{g}: a_{g} \in A\right\} \quad \text { and } B \rtimes_{\beta} G=\left\{\sum_{g \in G} b_{g} v_{g}: b_{g} \in B\right\}
$$

be crossed products of $\mathrm{C}^{*}$-algebras, where $u_{g}$ and $v_{g}$ are implementing unitaries.

Let $X \rtimes_{\gamma} G$ be the direct sum of $n$-copies of $X$ as a vector space, whose elements are written as formal sums so that

$$
X \rtimes_{\gamma} G=\left\{\sum_{g \in G} x_{g} w_{g}: x_{g} \in X\right\}
$$

where $w_{g}$ are indeterminates. We define the two sided actions of $A \rtimes_{\alpha} G$ and $B \rtimes_{\beta} G$ on $X \rtimes_{\gamma} G$ by

$$
\left(a u_{g}\right)\left(x w_{g^{\prime}}\right)=\left(a \gamma_{g}(x)\right) w_{g g^{\prime}} \quad \text { and } \quad\left(x w_{g^{\prime}}\right)\left(b v_{g}\right)=x \beta_{g^{\prime}}(b) w_{g^{\prime}} .
$$

We can define two sided inner products on $X \rtimes_{\gamma} G$ extending linearly the following values for monomials:

$$
\begin{aligned}
& A \rtimes_{\alpha} G\left\langle x_{1} w_{g_{1}}, x_{2} w_{g_{2}}\right\rangle={ }_{A}\left\langle x_{1}, \gamma_{g_{1} g_{2}^{-1}}\left(x_{2}\right)\right\rangle u_{g_{1} g_{2}^{-1}}, \\
& \left\langle x_{1} w_{g_{1}}, x_{2} w_{g_{2}}\right\rangle_{B \rtimes_{\beta} G}=\beta_{g_{1}^{-1}}\left(\left\langle x_{1}, x_{2}\right\rangle_{B}\right) v_{g_{1}^{-1} g_{2}} .
\end{aligned}
$$


Lemma 1.5. The $A \rtimes_{\alpha} G-B \rtimes_{\beta} G$ bimodule $X \rtimes_{\gamma} G$ satisfies the conditions from (1) to (8) in Lemma 1.3.

Proof. Every condition is checked by direct computations. For example (2),(3) and (8) are satisfied as follows.

(2):

$$
\begin{aligned}
& \left({ }_{A \rtimes_{\alpha} G}\left\langle x_{1} w_{g_{1}}, x_{2} w_{g_{2}}\right\rangle\right)^{*}=\left({ }_{A}\left\langle x_{1}, \gamma_{g_{1} g_{2}^{-1}}\left(x_{2}\right)\right\rangle u_{g_{1} g_{2}^{-1}}\right)^{*} \\
= & u_{g_{2} g_{1}^{-1} A}\left\langle\gamma_{g_{1} g_{2}^{-1}}\left(x_{2}\right), x_{1}\right\rangle={ }_{A}\left\langle x_{2}, \gamma_{g_{2} g_{1}^{-1}}\left(x_{1}\right)\right\rangle u_{g_{2} g_{1}^{-1}}={ }_{A \rtimes_{\alpha} G}\left\langle x_{2} w_{g_{2}}, x_{1} w_{g_{1}}\right\rangle,
\end{aligned}
$$

$$
A \rtimes_{\alpha} G\left\langle a\left(x_{1} w_{g_{1}}\right), x_{2} w_{g_{2}}\right\rangle={ }_{A}\left\langle a x_{1}, \gamma_{g_{1} g_{2}^{-1}}\left(x_{2}\right)\right\rangle u_{g_{1} g_{2}^{-1}}=a_{A \rtimes_{\alpha} G}\left\langle x_{1} w_{g_{1}}, x_{2} w_{g_{2}}\right\rangle
$$

and

$$
\begin{aligned}
& A \rtimes_{\alpha} G\left\langle u_{g}\left(x_{1} w_{g_{1}}\right), x_{2} w_{g_{2}}\right\rangle={ }_{A}\left\langle\gamma_{g}\left(x_{1}\right) w_{g g_{1}}, x_{2} w_{g_{2}}\right\rangle \\
= & { }_{A}\left\langle\gamma_{g}\left(x_{1}\right), \gamma_{g_{1} g_{2}^{-1}}\left(x_{2}\right)\right\rangle u_{g g_{1} g_{2}^{-1}}=u_{g A \rtimes_{\alpha} G}\left\langle x_{1} w_{g_{1}}, x_{2} w_{g_{2}}\right\rangle .
\end{aligned}
$$

We show the first part of (8).

$$
\begin{aligned}
& \left\langle a\left(x_{1} w_{g_{1}}\right), x_{2} w_{g_{2}}\right\rangle_{B \rtimes_{\beta} G}=\beta_{g_{1}^{-1}}\left(\left\langle a x_{1}, x_{2}\right\rangle_{B}\right) v_{g_{1}^{-1} g_{2}} \\
= & \beta_{g_{1}^{-1}}\left(\left\langle x_{1}, a^{*} x_{2}\right\rangle_{B}\right) v_{g_{1}^{-1} g_{2}}=\left\langle x_{1} w_{g_{1}}, a^{*}\left(x_{2} w_{g_{2}}\right)\right\rangle_{B \rtimes_{\beta} G}
\end{aligned}
$$

and

$$
\begin{aligned}
& \left\langle u_{g}\left(x_{1} w_{g_{1}}\right), x_{2} w_{g_{2}}\right\rangle_{B \rtimes_{\beta} G}=\left\langle\gamma_{g}\left(x_{1}\right) w_{g g_{1}}, x_{2} w_{g_{2}}\right\rangle_{B \rtimes_{\beta} G} \\
= & \beta_{g_{1}^{-1} g^{-1}}\left(\left\langle\gamma_{g}\left(x_{1}\right), x_{2}\right\rangle_{B}\right) v_{g_{1}^{-1} g g_{2}}=\beta_{g_{1}^{-1}}\left(\left\langle x_{1}, \gamma_{g^{-1}}\left(x_{2}\right)\right\rangle_{B}\right) v_{g_{1}^{-1} g^{-1} g_{2}} \\
= & \left\langle x_{1} w_{g_{1}}, \gamma_{g^{-1}}\left(x_{2}\right) w_{g^{-1} g_{2}}\right\rangle_{B \rtimes_{\beta} G}=\left\langle x_{1} w_{g_{1}}, u_{g^{-1}}\left(x_{2} w_{g_{2}}\right)\right\rangle_{B \rtimes_{\beta} G} .
\end{aligned}
$$

The other identities hold similarly.

Let $\left\{u_{i}\right\}_{i}$ be a finite basis of ${ }_{A} X$ and $\left\{v_{j}\right\}_{j}$ be a finite basis of $X_{B}$.

Lemma 1.6. Under the above situation, $\left\{u_{i} w_{e}\right\}_{i}$ constitutes a basis for ${ }_{A \rtimes_{\alpha} G} X$

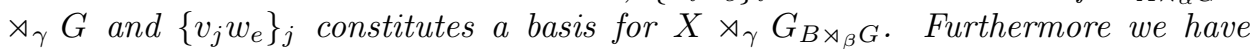
$\mathrm{r}-\operatorname{Ind}\left[X \rtimes_{\gamma} G\right]=\mathrm{r}-\operatorname{Ind}[X] u_{e}$ and $\mathrm{l}-\operatorname{Ind}\left[X \rtimes_{\gamma} G\right]=\mathrm{l}-\operatorname{Ind}[X] v_{e}$.

Proof. We only state the proof for r-Ind. For $x w_{g} \in X \rtimes_{\gamma} G$,

$$
\begin{aligned}
& \sum_{i} A \rtimes_{\alpha} G\left\langle x w_{g}, u_{i} w_{e}\right\rangle u_{i} w_{e}=\sum_{i} A\left\langle x, \gamma_{g}\left(u_{i}\right)\right\rangle u_{g} u_{i} w_{e} \\
= & \sum_{i}\left\langle x, \gamma_{g}\left(u_{i}\right)\right\rangle \gamma_{g}\left(u_{i}\right) w_{g}=\sum_{i} \gamma_{g}\left({ }_{A}\left\langle\gamma_{g^{-1}}(x), u_{i}\right\rangle u_{i}\right) w_{g} \\
= & \gamma_{g}\left(\sum_{i}{ }_{A}\left\langle\gamma_{g^{-1}}(x), u_{i}\right\rangle u_{i}\right) w_{g}=\gamma_{g}\left(\gamma_{g^{-1}}(x)\right) w_{g}=x w_{g} .
\end{aligned}
$$

Moreover,

$$
\mathrm{r}-\operatorname{Ind}\left[X \rtimes_{\gamma} G\right]=\sum_{i}{ }_{A}\left\langle u_{i} w_{e}, u_{i} w_{e}\right\rangle=\mathrm{r}-\operatorname{Ind}[X] u_{e} .
$$


Proposition 1.7. Let $X$ be a Hilbert $C^{*}$-bimodule of finite type, $G$ a finite group and $(X, A, B, G, \gamma, \alpha, \beta)$ a $G$-equivariant system. Then $X \rtimes_{\gamma} G$ is a Hilbert $C^{*}$ bimodule of finite type. In particular if $X$ is an imprimitivity bimodule, then $X \rtimes_{\gamma} G$ is also an imprimitivity bimodule.

Proof. This follows from Lemma 1.3, Lemma 1.5 and Lemma 1.6. In particular it is enough to recall that $X$ is an imprimitivity bimodule if and only if $\operatorname{r-Ind}[X]=I$, l-Ind $[X]=I$ by $[\mathrm{KW}$, Corollary 1.28].

Next we consider the case of a countable discrete group $G$. We denote by $X \rtimes_{f, \gamma} G$ the set of finite linear combinations $\left\{\sum_{g \in G} x_{g} w_{g}\right\}$. By the arguments in the finite group case, the properties (1)-(8) in Lemma 1.3 hold for the system $\left(X \rtimes_{f, \gamma}, A \rtimes_{f, \alpha}\right.$ $\left.G, B \rtimes_{f, \beta} G\right)$. For the completion and the continuities of two sided actions, we need the following discussions.

Proposition 1.8. Let $X$ be a Hilbert $C^{*}$-bimodules of finite type, $G$ a countable discrete group and $(X, A, B, G, \gamma, \alpha, \beta)$ a $G$-equivariant system. Then there exists a Banach space completion $X \rtimes_{\gamma} G$ of $X \rtimes_{f, \gamma} G$, which is a Hilbert $A \rtimes_{\alpha} G-$ $B \rtimes_{\beta} G$ bimodule of finite type. Moreover, we have $1-\operatorname{Ind}\left[X \rtimes_{\gamma} G\right]=1-\operatorname{Ind}[X] u_{e}$ and $\mathrm{r}-\operatorname{Ind}\left[X \rtimes_{\gamma} G\right]=\mathrm{r}-\operatorname{Ind}[X] u_{e}$.

Proof. Let $X={ }_{A} X$. Then $X$ is an $A-{ }_{A} \mathbf{K}\left({ }_{A} X\right)$ imprimitivity bimodule. There exists an action $\tilde{\beta}$ of $G$ on ${ }_{A} \mathbf{K}\left({ }_{A} X\right)$ such that $\tilde{\beta}_{g}\left(\Theta_{x, y}\right)=\Theta_{\gamma_{g}(x), \gamma_{g}(y)}$. By $[\mathrm{KW}$, Lemma 1.26], there exists an operator valued weight $E$ from ${ }_{A} \mathbf{K}\left({ }_{A} X\right)$ to $B$ such that $E\left(\Theta_{x, y}\right)=\langle x, y\rangle_{B}$. Then we have $E \tilde{\beta}_{g}(x)=\beta_{g}(E(x))$. We put $F(x)=$ $E(I)^{-1 / 2} E(x) E(I)^{-1 / 2}$ for $x \in{ }_{A} \mathbf{K}\left({ }_{A} X\right)$. Then $F$ is a conditional expectation from ${ }_{A} \mathbf{K}\left({ }_{A} X\right)$ to $B$ and satisfies the following:

$$
\begin{aligned}
\beta_{g}(F(x)) & =\beta_{g}\left(E(I)^{-1 / 2} E(x) E(I)^{-1 / 2}\right) \\
& =\left(\beta_{g}(E(I))\right)^{-1 / 2} \beta_{g}(E(x))\left(\beta_{g}(E(I))\right)^{-1 / 2} \\
& =E(I)^{-1 / 2} E\left(\tilde{\beta}_{g}(x)\right) E(I)^{-1 / 2}=F\left(\tilde{\beta}_{g}(x)\right) .
\end{aligned}
$$

By Theorem 1 in [CMW], $A \rtimes_{\alpha} G$ and ${ }_{A} \mathbf{K}\left({ }_{A} X\right) \rtimes_{\tilde{\beta}} G$ are strongly Morita equivalent by an imprimitivity bimodule $X \rtimes_{\gamma} G$ which is a completion of $X \rtimes_{\gamma, f} G$ in a linking algebra described as in [CMW]. By $[\mathrm{Kh}]$, there exists a conditional expectation $\tilde{F}$ from ${ }_{A} \mathbf{K}\left({ }_{A} X\right) \rtimes_{\tilde{\beta}} G$ to $B \rtimes_{\beta} G$ such that $\operatorname{Ind}[F]=\operatorname{Ind}[\tilde{F}]$. Since $X$ is of index finite type, $\operatorname{Ind}[F]$ is finite and equal to $\operatorname{Ind}[E]$ by $[\mathrm{KW}]$. Thus ${ }_{A} \mathbf{K}\left({ }_{A} X\right) \rtimes_{\tilde{\beta}} G$ is a Hilbert ${ }_{A} \mathbf{K}\left({ }_{A} X\right) \rtimes_{\tilde{\beta}} G-B \rtimes_{\beta} G$ bimodule of finite type. Consider the tensor product of $A \rtimes_{\alpha} G-{ }_{A} \mathbf{K}\left({ }_{A} X\right) \rtimes_{\tilde{\beta}} G$ bimodule $X \rtimes_{\gamma} G$ and ${ }_{A} \mathbf{K}\left({ }_{A} X\right) \rtimes_{\tilde{\beta}} G-B \rtimes_{\beta} G$ bimodule ${ }_{A} \mathbf{K}\left({ }_{A} X\right) \rtimes_{\tilde{\beta}} G$ of finite type. Then the properties in Definition 1.1 and Definition 1.2 hold for the completed triple $\left(X \rtimes_{\gamma} G, A \rtimes_{\alpha} G, B \rtimes_{\beta} G\right)$.

Remark. The same statements as in Proposition 1.8 hold for the reduced crossed products.

Let $(X, A, B, \gamma, \alpha, \beta, G)$ be a $G$-equivariant system. Moreover, we assume that $G$ is finite and abelian. We define the dual actions $\hat{\gamma}$ on $X \rtimes_{\gamma} G$ as follows:

$$
\hat{\gamma}_{\xi}\left(\sum_{g \in G} x_{g} w_{g}\right)=\sum_{g \in G}\langle\xi, g\rangle x_{g} w_{g} .
$$


Let $\hat{\alpha}, \hat{\beta}$ be the dual actions of $\alpha$ and $\beta$. Let $K$ be the matrix algebra on $l^{2}(G)$. The following is the bimodule version of Takesaki-Takai duality theorem:

Proposition 1.9. Let $X$ be a Hilbert $C^{*}$-bimodule of finite type, $G$ a abelian finite group and $(X, A, B, \gamma, \alpha, \beta, G)$ a $G$-equivariant system. Then

$$
\left(X \rtimes_{\gamma} G, A \rtimes_{\alpha} G, B \rtimes_{\beta} G, \hat{\gamma}, \hat{\alpha}, \hat{\beta}, \hat{G}\right)
$$

is a $\hat{G}$-equivariant system and we have the following isomorphism as Hilbert $C^{*}$ bimodules:

$$
\left(A \rtimes_{\alpha} G\right) \rtimes_{\hat{\alpha}} \hat{G}\left(\left(X \rtimes_{\gamma} G\right) \rtimes_{\hat{\gamma}} \hat{G}\right)_{\left(B \rtimes_{\beta} G\right) \rtimes_{\hat{\beta}} \hat{G}} \simeq A \otimes K(X \hat{\otimes} K)_{B \otimes K}
$$

where $X \hat{\otimes} K$ is the outer tensor product of two Hilbert $C^{*}$ bimodules ${ }_{A} X_{B}$ and ${ }_{K} K_{K}$.

Proof. The proof is similar to the case of crossed products of $\mathrm{C}^{*}$ and $\mathrm{W}^{*}$-algebras. We consider elements of second crossed products as $X$-valued (respectively $A$ valued and $B$-valued) functions on $G \times \hat{G}$. By the Fourier transform about the argument $\hat{G}$, the second crossed products are isomorphic to the $(A \otimes C(G)) \rtimes_{\alpha \otimes \rho} G$, $(X \otimes C(G)) \rtimes_{\gamma \otimes \rho} G$ and $(B \otimes C(G)) \rtimes_{\beta \otimes \rho} G$, where $\rho$ is the right translation action of $G$ on $C(G)$. Furthermore consider the following maps:

$$
\begin{gathered}
W: X \otimes C(G) \rightarrow X \otimes C(G) \quad \text { given by } \quad W\left(x \otimes \delta_{g}\right)=\gamma_{g}(x) \otimes \delta_{g}, \\
U: A \otimes C(G) \rightarrow A \otimes C(G) \quad \text { given by } \quad U\left(a \otimes \delta_{g}\right)=\alpha_{g}(a) \otimes \delta_{g}, \\
V: B \otimes C(G) \rightarrow B \otimes C(G) \quad \text { given by } \quad V\left(b \otimes \delta_{g}\right)=\beta_{g}(b) \otimes \delta_{g} .
\end{gathered}
$$

Then we have that $W(\gamma \otimes \rho) W^{-1}=i d \otimes \rho, \quad U(\alpha \otimes \rho) U^{-1}=i d \otimes \rho, V(\beta \otimes \rho) V^{-1}=$ $i d \otimes \rho$. Therefore the second crossed products are isomorphic to the above outer tensor products. It is easily checked that the resulting isomorphism is the one in the sense of Hilbert $\mathrm{C}^{*}$-bimodules.

\section{Categorical structure}

We consider two conditions on an action $\gamma$ of $G$ on $X$ for irreducibility of the crossed product $X \rtimes_{\gamma} G$.

Definition 2.1. Let $(X, A, B, G, \gamma, \alpha, \beta)$ be a $G$-equivariant system. Then the action $\gamma$ is called free if for $g \in G, g \neq e$, the following holds: If $T \in \mathrm{C}_{\mathbf{C}} \operatorname{End}_{\mathbf{C}}(X)$ satisfies $T(a x)=a T(x)$ and $T(x b)=T(x) \beta_{g}(b)$ for all $x \in X, a \in A$ and $b \in A$, then $T=0$.

The action $\gamma$ is called ergodic if for every $T \in{ }_{A} \operatorname{End}_{B}\left({ }_{A} X_{B}\right)$, the following holds: If $\gamma_{g} T=T \gamma_{g}$ for all $g \in G$, then $T \in \mathbf{C} \cdot I$.

Let $X g$ be a Hilbert $A$ - $B$ bimodule twisting the right $B$ action and right $B$-inner product by the automorphism $\beta_{g}$ of $B$ putting

$$
x \cdot b=x \beta_{g}(b) \text { and }\langle x, y\rangle_{B}=\beta_{g}^{-1}\left(\langle x, y\rangle_{B}\right)
$$

Then we remark that $\gamma$ is free if and only if for $g \neq e X$ and $X g$ are disjoint as Hilbert $A$ - $B$ bimodule.

Theorem 2.2. Let $X$ be a Hilbert $C^{*}$-bimodule of finite type, $G$ a finite group and $(X, A, B, G, \gamma, \alpha, \beta)$ a $G$-equivariant system. If $\gamma$ is free, then

$$
A \rtimes_{\alpha} G \operatorname{End}_{A \rtimes_{\beta} G}\left(X \rtimes_{\gamma} G\right)
$$


is isomorphic to the fixed point subalgebras of ${ }_{A} \operatorname{End}_{B}\left({ }_{A} X_{B}\right)$ under the action $\operatorname{ad}\left(\gamma_{g}\right)$ of $G$. Furthermore, if the action $\gamma$ is ergodic, then $X \rtimes_{\gamma} G$ is an irreducible bimodule.

Proof. We assume that $\gamma$ is free. Let $T \in_{A \rtimes_{\alpha} G} \operatorname{End}_{B \rtimes_{\beta} G}\left(X \rtimes_{\gamma} G\right)$. For all $g \in G$, we define an operator $T_{g} \in{ }_{\mathbf{C}} \operatorname{End}_{\mathbf{C}}(X)$ by the following formula:

$$
T\left(x w_{e}\right)=\sum_{g \in G} T_{g}(x) w_{g} .
$$

Since $G$ is finite, $T_{g}$ 's are well defined. We show that for $g \neq e, T_{g}=0$ holds. By $T\left(a\left(x w_{e}\right)\right)=a T\left(x w_{e}\right)$, we have $T_{g}(a x)=a T_{g}(x)$. On the other hand, by $T\left(\left(x w_{e}\right) b\right)=T\left(x w_{e}\right) b$, we have $T_{g}(x b)=T_{g}(x) \beta_{g}(b)$. Since $\gamma$ is free, for $g \neq e$ we have $T_{g}=0$. This shows that $T\left(x w_{e}\right)=T_{e}(x) w_{e}$.

From the right $G$-intertwining property,

$$
T\left(x w_{g}\right)=T\left(x w_{e} v_{g}\right)=T\left(x w_{e}\right) v_{g}=T_{e}(x) w_{e} v_{g}=T_{e}(x) w_{g} .
$$

This shows that

$$
T\left(\sum_{g \in G} x_{g} w_{g}\right)=\sum_{g \in G} T_{e}\left(x_{g}\right) w_{g} .
$$

At last, from the left $G$-intertwining property, we have $T\left(u_{g}\left(x w_{e}\right)\right)=u_{g} T\left(x w_{e}\right)$. This implies that

$$
T_{e}\left(\gamma_{g}(x)\right) w_{g}=\gamma_{g} T_{e}(x) w_{g} .
$$

This shows the first part. If $\gamma$ is moreover ergodic, then the fixed point subalgebra consists of only scalars, so $X \rtimes_{\gamma} G$ is irreducible.

Assume that the centers $Z(A)$ and $Z(B)$ of $A$ and $B$ consist of only scalars. The Hilbert $\mathrm{C}^{*}$-bimodule of finite type $X={ }_{A} X_{B}$ is called minimal if there exist two positive constants $C_{1}$ and $C_{2}$ such that

$$
C_{1} \sum_{i}{ }_{A}\left\langle T u_{i}, u_{i}\right\rangle=C_{2} \sum_{j}\left\langle v_{j}, T v_{j}\right\rangle_{B}
$$

hold for $T \in{ }_{A} \operatorname{End}_{B}(X)[\mathrm{KW}]$. Let $(X, A, B, \gamma, \alpha, \beta, G)$ be a $G$-equivariant system. $X={ }_{A} X_{B}$ is called $G$-minimal if the above condition holds for only $T \in$ ${ }_{A} \operatorname{End}_{B}(X)^{G}$.

Proposition 2.3. Under the above situation, let $\gamma$ be free and $X$ be $G$-minimal. Then $X \rtimes_{\gamma} G$ is also minimal.

Proof. We show that the crossed product system satisfies the condition of minimality. By the freeness of $\gamma,{ }_{A \rtimes_{\alpha} G} \operatorname{End}_{A \rtimes_{\beta} G}\left(X \rtimes_{\gamma} G\right)$ is isomorphic to the fixed point subalgebras of ${ }_{A} \operatorname{End}_{B}\left({ }_{A} X_{B}\right)$ under the action $\operatorname{ad}\left(\gamma_{g}\right)$ of $G$. By this the above condition is satisfied for the base $\left\{u_{i} \otimes I\right\}_{i}$ and $\left\{v_{j} \otimes I\right\}_{j}$.

Let $\bar{X}$ be the conjugate bimodule of $X$ as in [KW]. We define the conjugate action $\bar{\gamma}$ of $\gamma$ by $\bar{\gamma}_{g}(\bar{x})=\overline{\gamma_{g}(x)}$ for $x \in X$.

Proposition 2.4. We have the following:

(1) If $\left(X_{1}, A, B, \gamma^{X_{1}}, \alpha^{X_{1}}, \beta^{X_{2}}, G\right)$ and $\left(X_{2}, A, B, \gamma^{X_{2}}, \alpha^{X_{2}}, \beta^{X_{2}}, G\right)$ are $G$-equivariant systems, then there exists an action $\gamma^{X_{1} \oplus X_{2}}$ on $X=X_{1} \oplus X_{2}$ such

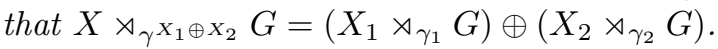

(2) $\overline{X \rtimes_{\gamma} G} \simeq \bar{X} \rtimes_{\bar{\gamma}} G$. 
(3) If ( $\left.X, A, B, \gamma^{X}, \alpha, \beta, G\right)$ and $\left(Y, B, C, \gamma^{Y}, \beta, \delta, G\right)$ are $G$-equivariant systems, then there exists an action $\gamma^{X \otimes Y}$ on $X \otimes_{B} Y$ such that

$$
\left(X \otimes_{B} Y\right) \rtimes_{\gamma^{X \otimes Y}} G \simeq\left(X \rtimes_{\gamma^{X}} G\right) \otimes_{B \rtimes_{\beta} G}\left(Y \rtimes_{\gamma^{Y}} G\right) .
$$

Proof. (1) is trivial. (2) We define a map $\varphi$ from $\overline{X \rtimes_{\gamma} G}$ to $\bar{X} \rtimes_{\bar{\gamma}} G$ by $\varphi\left(\overline{x w_{g}}\right)=$ $\overline{\gamma_{g^{-1}}(x)} w_{g^{-1}}$. This $\varphi$ gives the isomorphism.

(3) We define a map $\varphi$ from $\left(X \rtimes_{\gamma^{X}} G\right) \otimes_{B \rtimes_{\beta} G}\left(Y \rtimes_{\gamma^{Y}} G\right)$ to $\left(X \otimes_{B} Y\right) \rtimes_{\gamma^{X} \otimes Y} G$ by $\varphi\left(x w_{g} \otimes y w_{g^{\prime}}\right)=\left(x \otimes \gamma_{g}^{Y}(y)\right) w_{g g^{\prime}}$. Then this $\varphi$ intertwines two sided actions and maps two sided inner products exactly. This $\varphi$ gives the desired isomorphism.

Corollary. Let $(X, A, B, \gamma, \alpha, \beta)$ be a G-equivariant system and $Y=X \rtimes_{\gamma} G$. Consider an action $\gamma^{X \otimes \bar{X} \otimes X \cdots}$ on $X \otimes \bar{X} \otimes X \cdots$ by tensoring. Then $Y \otimes \bar{Y} \otimes Y \otimes$ $\bar{Y} \cdots$ is isomorphic to $(X \otimes \bar{X} \otimes X \otimes \bar{X} \cdots) \rtimes_{\gamma^{X} \otimes \bar{X} \otimes X \cdots G} G$.

Definition 2.5. The action $\gamma$ of $G$ on $X$ is strongly outer if all $\gamma^{X \otimes \bar{X} \otimes X \cdots \text { 's are }}$ free.

The above definition is a bimodule version of a strongly outer action in [CK].

Proposition 2.6. Under the same situation, if the action $\gamma$ is strongly outer, then the rule of the tensor power of $Y$ is isomorphic to the G-ergodic fusion rule of the tensor power of $X$.

Proof. This follows from Theorem 2.2 and Proposition 2.4.

The $G$-ergodic fusion rule is described as follows: We multiply minimal $G$ invariant bimodules and decompose them into the direct sum of minimal $G$-invariant bimodules.

\section{EXAMPLES}

In this section, we present some interesting examples of the crossed product bimodules.

Example 1. Let $M$ be a von Neumann algebra. Then $X=M$ is obviously a Hilbert $M-M$ bimodule. Let $G$ be a finite group, and $\alpha$ an action of $G$ on $M$. Put $\alpha=\beta=\gamma$. Then $(X, M, M, G, \gamma, \alpha, \beta)$ is a $G$-equivariant system. We note that $M \rtimes_{\gamma} G-M \rtimes_{\gamma} G$ bimodule $X \rtimes_{\gamma} G$ is irreducible if and only if $M \rtimes_{\gamma} G$ is a factor. And $\gamma$ is free if and only if $\alpha$ is freely acting in the sense of Kallman. Furthermore, $\gamma$ is ergodic if and only if $\alpha$ is ergodic on the center of $M$. Therefore the assumption in Theorem 2.2 corresponds with the usual condition which implies the factorness of $M \rtimes_{\alpha} G$.

Example 2. Let $G$ be a finite group, $A$ be a unital $\mathrm{C}^{*}$-algebra, $\delta$ be a properly outer action of $G$ on $A$ and $\pi$ be a unitary representation of $G$ on $\mathbf{C}^{n}$. Let $X=$ $A \otimes \mathbf{C}^{n}$. We make $X$ into a Hilbert $A$ - $A$ bimodule as follows.

$$
\begin{array}{rlrl}
a^{\prime}(a \otimes v) & =\left(a^{\prime} a\right) \otimes v, \quad & (a \otimes v) a^{\prime \prime}=\left(a a^{\prime \prime}\right) \otimes v, \\
A_{A}\left\langle a_{1} \otimes v_{1}, a_{2} \otimes v_{2}\right\rangle & =a_{1} a_{2}{ }^{*}\left\langle v_{1}, v_{2}\right\rangle, \quad\left\langle a_{1} \otimes v_{1}, a_{2} \otimes v_{2}\right\rangle_{A}=a_{1}{ }^{*} a_{2}\left\langle v_{2}, v_{1}\right\rangle .
\end{array}
$$

We define the $G$ actions on $A$ and $X$ as follows.

$$
\alpha_{g}(a)=\delta_{g}(a), \quad \beta_{g}(a)=\delta_{g}(a), \quad \gamma_{g}(a \otimes v)=\delta_{g}(a) \otimes \pi_{g}(v) .
$$

We denote this bimodule by $V(\pi)$.

At first we state simple categorical properties. 
Lemma 3.1. Let $\pi$ and $\rho$ be two finite dimensional unitary representations of a finite group $G$. We denote $\bar{\pi}$ as the conjugate representation of $\pi$. Then the followings hold.

(1) $V(\pi) \otimes V(\rho) \simeq V(\pi \otimes \rho)$.

(2) $\overline{V(\pi)} \simeq V(\bar{\pi})$.

Proof. We omit the proofs.

Lemma 3.2. The action $\gamma$ on $V(\pi)$ is free.

Proof. ${ }_{A} V(\pi)_{A}$ is isomorphic to the finite direct sum of ${ }_{A} A_{A}$ and ${ }_{A} V(\pi) g_{A}$ is isomorphic to the finite direct sum of ${ }_{A} A g_{A}$. They are disjoint since the action $\delta$ on $A$ is properly outer.

Proposition 3.3. Under the above situation, $A \rtimes_{\delta} G \operatorname{End}_{A \rtimes_{\delta} G}\left(V(\pi) \rtimes_{\gamma} G\right)$ is isomorphic to the commutant algebra of $\pi(G)$ in $M_{n}(\mathbf{C})$. Moreover, if $\pi$ is irreducible, the action $\gamma$ is free and ergodic.

Proof. ${ }_{A} \operatorname{End}_{A}(V(\pi))$ is isomorphic to $M_{n}(\mathbf{C})$ and $\operatorname{ad}\left(\gamma_{g}\right)$ on this $\mathrm{C}^{*}$-algebra is transformed into ad $\left(\pi_{g}\right)$. The fixed point algebra is exactly the commutant algebra of $\pi$.

Proposition 3.4. Under the same situation, the fusion rule of $X \otimes \bar{X} \otimes X \otimes \bar{X} \cdots$ is isomorphic to the decomposition rule of $\pi \otimes \bar{\pi} \otimes \pi \otimes \bar{\pi} \cdots$.

Proof. This follows from Lemma 3.1, Lemma 3.2 and Proposition 3.3.

Example 3. Let $R$ be a hyperfinite $\mathrm{II}_{1}$ factor and $S_{n}$ the symmetric group on $n$ letters. Put $a=(1,2,3) \in S_{3}, b=(1,2) \in S_{2} \subset S_{3}$. Let $A \simeq \mathbf{Z}_{3}$ be the cyclic group generated by $a$. Let $\mu: S_{3} \rightarrow$ Aut $R$ be an outer action. Put $P=R \rtimes_{\mu} S_{3} \supset$ $Q=R \rtimes_{\mu} S_{2}$, and $M=R \rtimes_{\mu} A \supset N=R$. Since $S_{3}=\left\{a^{n} b^{m}: n=1,2,3, m=\right.$ $1,2\} \simeq A \rtimes \mathbf{Z}_{2}$, there exists on outer automorphism $\sigma$ on $M$ such that $\sigma(N)=N$, $\sigma^{2}=i d$ and

$$
P \simeq M \rtimes_{\sigma} \mathbf{Z}_{2} \supset Q \simeq N \rtimes_{\sigma} \mathbf{Z}_{2} .
$$

The principal graph of the inclusion $N \subset M$ is the Dynkin diagram $D_{4}$ and the principal graph of the inclusion $Q \subset P$ is $A_{5}$.

We denote an element $x \in R \rtimes_{\mu} S_{3}$ by $x=\sum_{g \in S_{3}} x_{g} \lambda_{g} \quad\left(x_{g} \in R\right)$. Put $X=$ $N \lambda_{a} \oplus N \lambda_{a^{2}}$. Then $X$ is the direct sum of two Hilbert $N-N$ bimodules twisting the right action and right inner product on $N$ by the automorphisms $\mu_{a}$ and $\mu_{a^{2}}$. Let $\alpha=\beta=\sigma: \mathbf{Z}_{2} \rightarrow$ Aut $N$ and $\gamma$ be given by $\gamma\left(x_{1} \lambda_{a} \oplus x_{2} \lambda_{a^{2}}\right)=\sigma\left(x_{2}\right) \lambda_{a} \oplus \sigma\left(x_{1}\right) \lambda_{a^{2}}$. Then $\left(X, N, N, \mathbf{Z}_{2}, \gamma, \alpha, \beta\right)$ is a $\mathbf{Z}_{2}$-equivariant system. We can check that the action $\gamma$ is free and ergodic. By Theorem 2.2, the crossed product $X \rtimes_{\gamma} \mathbf{Z}_{2}$ is an irreducible Hilbert $Q-Q$ bimodule, where $Q=N \rtimes_{\sigma} \mathbf{Z}_{2}$. Moreover the Hilbert $Q$ - $Q$ bimodule $X \rtimes_{\gamma} \mathbf{Z}_{2}$ represents the middle vertex of the principal graph $A_{5}$.

The $\mathbf{Z}_{2}$ action $\sigma$ induces flipping the two tails of $D_{4}$ and the restriction $\gamma$ of $\sigma$ to the invariant bimodule $X$ provides a good example of free and ergodic action in the sense of this paper. The dual action $\hat{\sigma}: \hat{\mathbf{Z}}_{2} \rightarrow \operatorname{Aut}(P, Q)$ is the orbifold action on $A_{5}$. We have many similar interesting examples as follows.

Example 4. Kawahigashi [Kaw] introduced the orbifold construction for subfactors to construct new subfactors as simultaneous fixed point algebras (or crossed 
products) by finite group actions. In particular, he considers a kind of duality between subfactors with the principal graphs of the Dynkin diagrams $A_{4 n-3}$ and $D_{2 n}$. The automorphisms appearing in the orbifold construction are non-strongly outer action of Choda-Kosaki [CK] or non-properly outer action of S. Popa [Po1].

Let $N \subset M$ be an inclusion of type $\mathrm{II}_{1}$ factors. Let $\alpha$ be an automorphism on $M$ and $\alpha M$ the $M-M$ bimodule obtained by $x \cdot \xi \cdot y=\alpha(x) \xi y$. Even if ${ }_{M}\left(M_{n}\right)_{M}$ contains $\alpha M, \alpha$ does not necessarily preserve $N$ globally. But if there exists $\beta \in$ Aut $N$ such that

$$
{ }_{M}\left(\alpha M \otimes_{M} M\right)_{N} \simeq{ }_{M}\left(M \otimes_{N} \beta N\right)_{N}
$$

then there exists a unitary $u \in M$ such that $\operatorname{Ad} u \circ \alpha$ preserves $N$ globally and $\beta=\left.\operatorname{Ad} u \circ \alpha\right|_{N}$. Such automorphisms are studied and characterized by M. Izumi [I1], [I2], H. Kosaki [Ko1], [Ko2], S. Goto [Go] and S. Yamagami [Y]. For example suppose that the principal graph is the Dynkin diagram $A_{4 n-3}$. Let $X_{0}-X_{1}-$ $X_{2}-\cdots-X_{4 n-4}$ be the $M-M$ bimodules corresponding to the principal graph $A_{4 n-3}$. We assume $X_{0}={ }_{M} M_{M}$ represents $*$. Since $\operatorname{dim} X_{0}=\operatorname{dim} X_{4 n-4}=1$, there exists an automorphism $\alpha \in$ Aut $M$ such that $X_{4 n-4} \simeq \alpha M$. They show that we can choose $\alpha \in$ Aut $M$ such that $\alpha(N)=N$ and $\alpha^{2}=i d$. Thus we can consider the simultaneous crossed products $N \rtimes_{\alpha} \mathbf{Z}_{2} \subset M \rtimes_{\alpha} \mathbf{Z}_{2}$. To modify their results to our setting, replace bimodules ${ }_{M} L^{2}(M)_{M}, L^{2}(M) \otimes_{N} L^{2}(M), \ldots$ by Hilbert C*-bimodules ${ }_{M} M_{M},{ }_{M}\left(M \otimes_{N} M\right)_{M}, \ldots$. In fact they become self dual Hilbert $\mathrm{W}^{*}$-bimodules. Their argument holds without any change. Then the restriction of the orbifold actions to invariant submodules provides many interesting examples of actions on Hilbert $\mathrm{C}^{*}$-bimodules.

\section{REFERENCES}

[Bl] B. Blackadar, K-Theory for operator algebras, MSRI Publications 5 (1986). MR 88g:46082

[Ch] M. Choda, Duality for finite bipartite graphs (with application to $\mathrm{II}_{1}$ factors), Pacific J. Math. 158 (1993), 49-65. MR 94e:46113

[CK] M. Choda and H. Kosaki, Strongly outer actions for an inclusion of factors, J. Funct. Anal. 122 (1994), 315-332. MR 96b:46085

[CKRW] D. Crocker, A. Kumjian, I. Raeburn and D. P. Williams, An equivariant Brauer group and actions of group $C^{*}$-algebras, J. Funct. Anal. 146 (1997), 171-184.

[CMW] R. E. Curto, P. S. Muhly and D. P. Williams, Cross products of strongly Morita equivalent $C^{*}$-algebras, Proc. AMS. 90 (1984), 528-530. MR 85i:46083

[Co] F. Combes, Crossed products and Morita equivalence, Proc. London Math. Soc. 49 (1984), 289-306. MR 86c:46081

[Go] S.Goto, Orbifold construction for Non-AFD subfactors, Internat. J. Math. 5 (1994), 725-746. MR 95h:46094

[I1] M. Izumi, Application of fusion rules to classification of subfactors, Publ. RIMS Kyoto Univ. 27 (1991), 953-994. MR 93b:46121

[I2] M.Izumi, On type II and type III principal graphs of subfactors, Math. Scand. 73 (1993), 307-319. MR 95a:46084

[Jo] V. Jones, Index for subfactors, Invent. Math. 72 (1983), 1-25. MR 84d:46097

[Kaj] T. Kajiwara, Remarks on strongly Morita equivalent $C^{*}$-crossed products, Math. Japon. 32 (1987), 257-260. MR 89h:46095

[Kas] G.G.Kasparov, K-theory, group $C^{*}$-algebras and higher signatures (Conspectus), Chernogolovka (1981).

[Kat] Y. Katayama Takesaki's duality on bipartite graph, preprint.

[Kaw] Y. Kawahigashi, On flatness of Ocneanu's connections on the Dynkin diagrams and classification of subfactors, J. Funct. Annal. 127 (1995), 63-107. MR 95j:46075 
[KaY] T. Kajiwara and S. Yamagami, Irreducible bimodules associated with crossed product algebras II, Pac. J. Math. 171 (1995), 209-229. MR 96m:46124

[Kh] M. Khoshkam, Hilbert $C^{*}$-modules and conditional expectations on crossed products, J.Austral. Math. Soc. 61 (1996), 106-118. CMP 96:16

[KW] T. Kajiwara and Y. Watatani, Jones index theory by Hilbert $C^{*}$-bimodules and K-theory, preprint.

[Ko1] H. Kosaki, Automorphisms in the irreducible decompositions of sectors, Quantum and Non Commutative Analysis, Kluwer Academic (1993), 305-316. MR 95e:46073

[Ko2] H. Kosaki, Sector theory and automorphisms for factor - subfactor pairs, J. Math. Soc. Japan 48 (1996), 427-454. MR 97e:46083

[KoY] H. Kosaki and S. Yamagami, Irreducible bimodules associated with crossed product algebras, Int. Math. J. (1992), 661-676. MR 94f:46087

[KRW] A. Kumjian, I. Raeburn and D. P. Williams, The equivariant Brauer groups of commuting free and proper actions are isomorphic, Proc. Amer. Math. Soc. 124 (1996), 809-817. MR 96f:46107

[Oc] A. Ocneanu, Quantized groups, string algebras, and Galois theory for algebra, in Operator Algebras and Applications. Vol II, London Mathematical Lecture Note Series Vol. 136, Cambridge Univ. Press, 1988, 119-172. MR 91k:46068

[Po1] S. Popa, On the classification of actions of amenable groups on subfactors, C.R. Acad. Sc. Paris. 315 (1992), 295-299. MR 93i:46110

[Po2] S. Popa, Classification of amenable subfactor of type II, Acta Math. 172 (1994), 163255. MR 95f:46105

[PP] M. Pimsner and S. Popa, Entropy and index for subfactors, Ann. Scient. Ec. Norm. Sup. 19 (1986), 57-106. MR 87m:46120

[Ri1] M. A. Rieffel, Induced representations of $C^{*}$-algebras, Adv. Math. 13 (1974), 176-257. MR 50:5489

[Ri2] M. A. Rieffel, Morita equivalence for $C^{*}$-algebras and $\mathrm{W}^{*}$-algebras, J. Pure Appl. Algebras 5 (1974), 51-96. MR 51:3912

[Ri3] M. A. Rieffel, Morita equivalence for operator algebras, Proc. Sympos. Pure Math. Amer. Math. Soc. 38, Part1 (1982), 285-298. MR 84k:46045

[Wa] Y. Watatani, Index for $C^{*}$-subalgebras, Memoir Amer. Math. Soc. 83, no. 424 (1990), 1-117. MR 90i:46104

[Y] S. Yamagami, Group symmetry in tensor categories, preprint.

Department of Environmental and Mathematical Sciences, Okayama University, TSushima, OKaYAma 700, JaPan

E-mail address: kajiwara@math.ems.okayama-4.ac.jp

Graduate School of Mathematics, Kyushu University, Ropponmatsu, Fukuoka, 810 JAPAN

E-mail address: watatani@rc.kyush-u.ac.jp 